\title{
Effect of Carbohydrate Intolerance and Gestational Diabetes on Obstetric and Perinatal Outcomes
}

\author{
Leticia Lara-Ávila*, José Manuel Brambila-Durán, Diego Armando Camacho-Ramírez, \\ Felipe de Jesús Peraza-Garay, Adrián Herrera-Salazar, Fred Morgan-Ortiz \\ Health Sciences Research and Teaching Center, Autonomous University of Sinaloa, Civil Hospital of Culiacan, \\ Culiacan, Mexico \\ Email: ${ }^{*}$ letylara79@hotmail.com
}

Received 1 February 2014; revised 1 March 2014; accepted 8 March 2014

Copyright (C) 2014 by authors and Scientific Research Publishing Inc.

This work is licensed under the Creative Commons Attribution International License (CC BY). http://creativecommons.org/licenses/by/4.0/

\section{(c) (i) Open Access}

\begin{abstract}
Objectives: To compare the frequency of obstetric and perinatal complications among patients with carbohydrate intolerance (CHI) and gestational diabetes (GD). Materials and Methods: In a prospective study at the Civil Hospital of Culiacán, we studied 182 pregnant patients between 16 and 30 weeks of gestation (WG) who received a glucose tolerance test following the oral administration of $100 \mathrm{~g}$ of glucose. The patients were classified as normal $(n=53)$, CHI $(n=61)$ and GD ( $n$ $=68$ ) between January 15 to November 30,2012 . The analyzed variables included the frequency of polyhydramnios, preeclampsia-eclampsia, obstetric hemorrhage, fetal macrosomia, and admission to a neonatal intensive care unit (NICU). Results: No significant differences were found between women with $\mathrm{CHI}$ and women with GD with respect to obstetric hemorrhage $(P=0.774)$, preeclampsia-eclampsia $(P=0.590)$, and macrosomia $(P=0.119)$. However, polyhydramnios was more frequent in $\mathrm{CHI}$ group $(\mathrm{OR}=3$ ) compared to $\mathrm{GD}$, whereas admission to the NICU was lower in the $\mathrm{CHI}$ group compared to GD group $(\mathrm{OR}=0.38)$. Conclusion: Preeclampsia-eclampsia, obstetric hemorrhage, and macrosomia were similar among patients with CHI and GD. In contrast, polyhydramnios was more frequent in patients with $\mathrm{CHI}$ compared to patients with GD, but admission to the NICU was more frequent in patients with GD.
\end{abstract}

\section{Keywords}

Glucose Tolerance Test; Carbohydrate Intolerance; Gestational Diabetes; Perinatal Outcome

\footnotetext{
"Corresponding author.
} 


\section{Introduction}

Gestational diabetes (GD) is the most common metabolic complication during pregnancy and is defined as any degree of glucose intolerance that is first seen or identified during pregnancy [1]. Internationally, the prevalence of GD is variable, with reports ranging from $1 \%$ to $14 \%$ of pregnancies based on the characteristics of the studied populations [2] [3]. Moreover, the development of GD may be attributed to differences in eating habits and the genetic factors of individual patients. In Mexico, the registered prevalence of GD is as high as $17 \%$ [4].

For many years, there has been a divergence with respect to the diagnosis of GD [5], and the most appropriate strategy for the screening and diagnosis of this disorder remains controversial [6]. Factors contributing to the lack of a unified diagnosis include 1) differences in the understanding of what is being detected, 2) differences in the proposed significance of GD, particularly after the publication of studies on hyperglycemia and its adverse effects on pregnancy (HAPO) [7], and 3) contrasting perspectives between endocrinologists, obstetricians, epidemiologists, and health economists [8].

The traditional manner for establishing a diagnosis of GD in Mexico is an oral glucose tolerance test (OGTT) following the administration of $100 \mathrm{~g}$ of glucose. This test uses a reference fasting value equal to or greater than $95 \mathrm{mg} / \mathrm{dl}, 180 \mathrm{mg} / \mathrm{dl}$ at 1 hour, $155 \mathrm{mg} / \mathrm{dl}$ at 2 hours, and $140 \mathrm{mg} / \mathrm{dl}$ at 3 hours. A $75 \mathrm{~g}$ glucose load may also be used, and this method uses the same reference values. The diagnosis of GD is confirmed when 2 or more altered glucose values are observed [9].

However, data related to patients with only a single altered value in the OGTT and their prognosis or management are limited, given that for many years, attention was completely focused on outlining the guidelines for the diagnosis, prognosis, and management of patients with a diagnosis of GD. Moreover, it has been reported that glucose intolerance (an altered value in the OGTT) seems to be an evolutionary process in the natural course of the disease towards diabetes [7], and there may also be a linear correlation between adverse perinatal outcomes, primarily fetal macrosomia, and an increase in the blood level of insulin C-peptide in umbilical cord blood [10]. The purpose of this work was to determine whether a single altered value in the glucose tolerance test following the administration of $100 \mathrm{~g}$ of glucose is associated with a frequency of maternal and perinatal complications similar to that of patients with GD.

\section{Materials and Methods}

Prior approval by the research and ethics committee of the Civil Hospital of Culiacan was obtained. From January 15 to November 30, 2012, screening tests were performed in 742 pregnant women at 24 - 28 weeks of gestation (WG) following the oral administration of $50 \mathrm{~g}$ of glucose to determine the 1-hour glycemic blood levels. Of the 742 patients who received $50 \mathrm{~g}$ of oral glucose, 182 patients demonstrated a positive result (equal to or greater than $130 \mathrm{mg}$ ) and underwent a $100 \mathrm{~g}$ OGTT no later than 1 week after the initial screening result was obtained. Before conducting the OGTT, the patients were asked to consume a regular diet on the day prior to the test, to fast after $10 \mathrm{pm}$, and to present for the test with no greater than 10 hours of fasting. The basal glucose level was obtained at 1, 2, and 3 hours after the $100 \mathrm{~g}$ glucose load. The following values were considered normal: $95 \mathrm{mg} / \mathrm{dl}$ after fasting, $180 \mathrm{mg} / \mathrm{dl}$ at 1 hour, $155 \mathrm{mg} / \mathrm{dl}$ at 2 hours, and $140 \mathrm{mg} / \mathrm{dl}$ at 3 hours. With respect to the results of the OGTT, 53 patients were classified as normal (no altered value in the OGTT), 61 patients were classified as having carbohydrate intolerance ( 1 altered value in the OGTT), and 68 patients were classified as having GD ( 2 or more altered values in the OGTT). Each patient was followed up accordingly during their prenatal care; normal patients received outpatient care in gynecology and obstetrics, and patients with carbohydrate intolerance and GD received prenatal care from the maternal medicine department in conjunction with nutrition and endocrinology. The information from these visits was gathered from the electronic records, and the outcomes were evaluated.

The following variables were analyzed for each group of patients: age, socioeconomic level, number of births, number of abortions, pre-gestational weight, body mass index (BMI), WG at the time of birth, frequency of obstetric hemorrhages (following a vaginal birth with a blood loss greater than $500 \mathrm{ml}$ or a C-section with a blood loss greater than $1000 \mathrm{ml}$ ), preeclampsia-eclampsia (arterial hypertension greater than 140/90 $\mathrm{mmHg}$ and proteinuria equal to or greater than $300 \mathrm{mg}$ in 24 hours), obstetric hysterectomy, and polyhydramnios (diagnosed via ultrasound considering the presence of a single pocket greater than $8 \mathrm{~cm}$ ). The following were among the evaluated perinatal results: the frequency of intrauterine growth restriction (IUGR) (diagnosed as an estimated fetal weight below the $10^{\text {th }}$ percentile with alterations in the Doppler or below the $3^{\text {rd }}$ percentile), fetal macrosomia 
(birth weight equal to or greater than 4000 g), Apgar score at 5 minutes $<7$, and admission to the neonatal intensive care unit (NICU).

The data were analyzed with the SPSS statistical package version 14.0, using the Chi-squared test for categorical variables and One-way Anova or Student's t-test for numerical variables. Odds ratios and their respective $95 \%$ confidence intervals were calculated to measure the strength of associations between variables.

\section{Results}

The sample consisted of a total of 182 pregnant patients from the Civil Hospital in Culiacan $(n=118)$ and the Sinaloa Women's Hospital $(n=64)$, and the demographic characteristics of these patients are listed in Table 1. In this study, $88.4 \%(n=161)$ of the patients who received an OGTT were overweight or obese, and of these, close to $70 \%$ demonstrated carbohydrate intolerance $(n=44)$ or $(n=68)$. In patients with GD, metformin was used in 22.1\% ( $n=15)$, and 29.4\% ( $n=20)$ required management with insulin.

With respect to vaginal birth or cesarean complications, the risk for an obstetric hemorrhage was greater in the group with carbohydrate intolerance (OR = 2.6; CI 95\%: 0.86 - 7.86; $p=0.090)$ and GD (OR = 3.2; CI 95\%: $1.09-9.35 ; p=0.033$ ) compared with the group of normal patients.

The risk of preeclampsia-eclampsia was significantly greater in patients with carbohydrate intolerance (OR = 7.94; CI 95\%: 2.53 - 24.87; $p=0.000$ ) or GD (OR = 10.26; CI 95\%: 3.33 - 31.62; $p=0.000)$ compared to normal patients. The risk of polyhydramnios in the group with carbohydrate intolerance (OR 33.7; CI 95\%: 4.3 260.5) or GD (OR = 11.1; CI 95\%: 1.4 - 88.7) was also greater than that observed in normal patients. The risks of adverse fetal outcomes, including macrosomia, an Apgar score $<7$, and admission to the NICU, were also greater in the carbohydrate intolerance and GD groups compared to patients with normal glucose levels (Table 2).

No significant differences were found in the group with carbohydrate intolerance compared to GD in relation to obstetric hemorrhage $(P=0.774)$ and preeclampsia-eclampsia $(P=0.590)$. However, the risk of polyhydramnios was greater in the carbohydrate intolerance group compared to GD (OR = 3.0; CI 95\%: $1.3-6.7)$. In relation

Table 1. General demographic characteristics of women based on the OGTT results.

\begin{tabular}{|c|c|c|c|c|}
\hline \multirow{2}{*}{ Characteristic } & \multicolumn{3}{|c|}{ Mean \pm Standard Deviation } & \multirow{2}{*}{$P$ Value } \\
\hline & Normal $(n=53)$ & Carbohydrate Intolerance $(n=61)$ & Gestational Diabetes $(n=68)$ & \\
\hline AGE (years) ${ }^{\&}$ & $27.08( \pm 7.14)$ & $28.66( \pm 6.4)$ & $27.90( \pm 6.6)$ & 0.455 \\
\hline Socioeconomic Status & & & & 0.0002 \\
\hline Middle & $71.7 \%(38)$ & $90.1 \%(55)$ & $66.2 \%(45)$ & \\
\hline Low & $28.3 \%(15)$ & $9.9 \%(6)$ & $33.8 \%(23)$ & \\
\hline No. of Pregnancies & & & & 0.000 \\
\hline 1 & $28.4 \%(15)$ & $32.8 \%(20)$ & $14.7 \%(10)$ & \\
\hline 2 & $35.8 \%(19)$ & 0 & $27.9 \%(19)$ & \\
\hline$\geq 3$ & $35.8 \%(19)$ & $67.2 \%(41)$ & $57.4 \%(39)$ & \\
\hline Abortion $^{\$}$ & $15.0 \%(8)$ & $16.3 \%(10)$ & $14.7 \%(10)$ & 0.345 \\
\hline Pregestational Weight (KGS) ${ }^{\&}$ & $78.8( \pm 10.5)$ & $75.2( \pm 7.5)$ & $81.0( \pm 9.6)$ & 0.002 \\
\hline $\mathbf{B M I}^{\$}$ & & & & 0.000 \\
\hline Normal (18.5 - 24.9) & $7.5 \%(4)$ & $27.9 \%(17)$ & 0 & \\
\hline Overweight (25 - 29.9) & $39.6 \%(21)$ & $27.9 \%(17)$ & $14.7 \%(10)$ & \\
\hline Obese $(>=30)$ & $52.8 \%(28)$ & $44.2 \%(27)$ & $85.3 \%(58)$ & \\
\hline Weeks of Gestation at Birth ${ }^{\&}$ & $38.76( \pm 1.01)$ & $38.12( \pm 1.04)$ & $37.6( \pm 0.8)$ & 0.000 \\
\hline
\end{tabular}

\& = One way ANOVA for comparison of means; \$ = Chi squared test for comparison of categorical variables. 
Table 2. Risk of maternal and perinatal complications between patients with carbohydrate intolerance, GD and a normal OGTT result.

\begin{tabular}{ccccccc} 
& Normal & \multicolumn{2}{c}{ Carbohydrate Intolerance } & \multicolumn{2}{c}{ Gestational Diabetes } & $P$ Value \\
& $\%(\mathrm{n})$ & $\%(\mathrm{n})$ & OR $^{*}$ & $\%(\mathrm{n})$ & OR $^{*}$ & 0.022 \\
\hline Obstetric Hemorrhage & $9.4 \%(5)$ & $21.3 \%(13)$ & $2.60(0.86-7.8)$ & $25.0 \%(17)$ & $3.20(1.1-9.3)$ & 0.000 \\
Preeclampsia-Eclampsia & $7.5 \%(4)$ & $39.3 \%(24)$ & $7.9(2.5-24.8)$ & $45.6 \%(31)$ & $10.2(3.3-31.6)$ & - \\
Obstetric Hysterectomy & $0 \%(0)$ & $3.3 \%(2)$ & - & $2.9 \%(2)$ & & 0.242 \\
Polyhydramnios & $1.8 \%(1)$ & $39.3 \%(24)$ & $33.7(4.3-260.5)$ & $17.6 \%(12)$ & $11.1(1.4-88.7)$ & 0.026 \\
IUGR & $1.9 \%(1)$ & $19.7 \%(12)$ & $12.73(1.6-10.1)$ & $27.9 \%(19)$ & $20.1(2.6-156.3)$ & 0.000 \\
Fetal Macrosomia & $7.5 \%(4)$ & $21.3 \%(13)$ & $3.3(1.01-10.9)$ & $35.3 \%(24)$ & $6.68(2.1-20.7)$ & 0.000 \\
Apgar $>7$ & $7.5 \%(4)$ & $8.2 \%(5)$ & $1.09(0.2-4.3)$ & $10.3 \%(7)$ & $1.4(0.3-5.0)$ & 0.058 \\
NICU & $7.5 \%(4)$ & $18.0 \%(11)$ & $2.7(0.8-9.0)$ & $36.8 \%(25)$ & $7.1(2.3-22.1)$ & 0.000 \\
\hline
\end{tabular}

*Compared with normal patients in the OGTT; IUGR: Intrauterine Growth Restriction; NICU: Neonatal Intensive Care Unit.

to the perinatal outcomes between patients with carbohydrate intolerance and GD, significant differences were found, with a lower risk of neonate admissions to the NICU among patients with CHI (OR 0.38; CI 95\%: 0.17 0.86) (Table 3).

\section{Discussion}

Given the relevance of GD with respect to adverse maternal and perinatal outcomes, controversy has arisen with regards to the diagnosis and management of this condition. Since 1998, diagnosis based on the glucose tolerance test curve following the oral administration of 100 or $75 \mathrm{~g}$ of glucose was reserved for patients with 2 or more altered values [11]. However, it was recently proposed at the international level to stop performing glucose screenings and to begin administering carbohydrate tolerance tests with a 75 g glucose load, moving the cut-off points to $92 \mathrm{mg} / \mathrm{dl}$ at 1 hour, $180 \mathrm{mg} / \mathrm{dl}$ at 2 hours, and $153 \mathrm{mg} / \mathrm{dl}$ at 3 hours. In addition, with this approach, a diagnosis of GD may be made with a single altered value [7]. This method was accepted by the American Diabetes Association (ADA) [12] but not by other international organizations such as the American College of Obstetricians and Gynecologists in the United States [13]. Here in Mexico, it was recently found that adopting the IADPSG criteria would result in a 3-fold increase in the number of patients with a GD diagnosis, which not only involves a psychological concern for the majority of patients but also represents an important increase in the cost of laboratory tests, nutrition, obstetricians, and endocrinology care. These factors may also represent a financial burden outside the reach of health care programs, especially in developing countries and Latin American countries in particular. Thus, it is important to conduct larger randomized clinical studies to evaluate the effect of the new ADA IADPSG criteria for the treatment of patients with a single altered value, not only in terms of the prevalence of GD but also in the associated adverse maternal, fetal, and neonatal outcomes and the health economics policies of many countries [14].

Based on the observed results in this study, the number of patients with carbohydrate intolerance and GD were very similar with respect to number and perinatal outcome, which is in agreement with the findings reported by Di Cianni et al. [6] in a study involving 4053 pregnant women with a positive glucose screening test who received a 3-hour OGTT with $100 \mathrm{~g}$ of glucose. These authors observed that elevated readings on the curve were not an isolated phenomenon. Rather, this previous study suggested that the pathophysiology in intolerant patients may represent a step prior to the development of GD, which is in line with the similarities in complications observed in both groups.

GD is known to be an important factor for the development of fetal macrosomia, and a previous study by Ortega et al. found that carbohydrate intolerance also increases the risk of macrosomia [15]. In our study, we observed similar development of macrosomia in the group of patients with carbohydrate intolerance and GD but significant differences between these groups and patients with normal OGTTs. 
Table 3. Risk of maternal and perinatal complications between patients with GD and carbohydrate intolerance.

\begin{tabular}{ccccc}
\hline \multirow{2}{*}{ Complications } & Carbohydrate Intolerance $n=61$ & Gestational Diabetes $n=68$ & OR (CI 95\%) & $P$ Value \\
\cline { 2 - 4 } & $\%(n)$ & $\%(n)$ & & 0.774 \\
\hline Obstetric Hemorrhage & $21.3 \%(13)$ & $25 \%(17)$ & $0.3-1.8)$ & 0.590 \\
Preeclampsia-Eclampsia & $39.3 \%(24)$ & $45.5 \%(31)$ & $3.0(1.3-6.7)$ & 0.010 \\
Polyhydramnios & $39.3 \%(24)$ & $17.6 \%(12)$ & $0.50(0.2-1.0)$ & 0.119 \\
Fetal Macrosomia & $21.3 \%(13)$ & $35.2 \%(24)$ & $0.78(0.2-2.5)$ & 0.915 \\
Apgar $>7$ & $8.2 \%(5)$ & $10.2 \%(7)$ & $0.38(0.1-0.8)$ & 0.029 \\
\hline Nicu & $18 \%(11)$ & $36.7 \%(25)$ &
\end{tabular}

Advanced maternal age also plays an important role in the development of GD. In a study including 22,571 patients, it was observed that maternal age greater than 40 years demonstrated the development of diabetes as the most frequent complication [16]. The study by Ortega et al. further showed that maternal age older than 37 years and a BMI equal to or greater to $36 \mathrm{~kg} / \mathrm{m}^{2}$ were related to a GD frequency of $83 \%$ and a carbohydrate intolerance rate of $17 \%$ [15]. In this study, age did not show any relationship with abnormal OGTT results. Similarly, this study also confirms the increased risk of carbohydrate metabolism alterations associated with overweight status or obesity.

The relationship between GD and adverse maternal factors, such as obstetric hemorrhage or preeclampsiaeclampsia, is well-established [7] [17]. The results of this study coincide with prior reports showing a greater preeclampsia risk in patients with GD and carbohydrate intolerance compared to patients with a normal OGTT.

We also found that the frequency of polyhydramnios was greater in the group of patients with carbohydrate intolerance (39.3\%) compared to patients with GD (17.3\%). This result differs from that published in other studies, where it has been reported that the frequency of polyhydramnios is greater in diabetics than in patients with carbohydrate intolerance [7].

The Apgar score of newborns of mothers with carbohydrate intolerance, GD, and a normal OGTT was similar at 1 and 5 minutes. However, the frequency of neonates admitted to the NICU was greater among patients with carbohydrate intolerance and GD compared to normal patients. This result agrees with previous reports in the literature, given that the children of diabetic and glucose-intolerant mothers have a higher risk of complications such as respiratory insufficiency syndrome, hypoglycemia, and hypocalcemia compared to the children of mothers without any alteration in carbohydrate metabolism [16]. The main causes for admission to the NICU for the patients in this study were respiratory insufficiency and hypoglycemia.

\section{Conclusion}

The results obtained in this study reveal similarities in adverse perinatal outcomes between patients with GD and carbohydrate intolerance, which reinforces the need to establish treatment and follow-up for patients with an altered value in the glucose tolerance test. The significance of this finding was also previously proposed by Landon et al. in 2009 as this study found that providing treatment to such patients did not significantly reduce the frequency of fetal or perinatal death but did reduce the risk of fetal macrosomia, shoulder dystocia, birth by cesarean, and hypertensive diseases of pregnancy [18].

\section{References}

[1] WHO (1999) Definition, Diagnosis and Classification of Diabetes Mellitus and Its Complications. Report of World Health Organization Consultation. WHO, Geneva.

[2] Meltzer, S.J., Snyder, J., Penrod, J.R., Nudi, M. and Morin, L. (2010) Gestational Diabetes Mellitus Screening and Diagnosis: A Prospective Randomized Controlled Trial Comparing Costs of One-Step and Two-Step Methods. BJOG, 117, 407-415. http://dx.doi.org/10.1111/j.1471-0528.2009.02475.x

[3] Jimenez-Moleon, J.J., Bueno-Cavanillas, A., Luna-Del-Castillo, J.D., Garcia-Martin, M., Lardelli-Claret, P. and Gal- 
vez-Vargas, R. (2002) Prevalence of Gestational Diabetes Mellitus: Variations Related to Screening Strategy Used. European Journal of Endocrinology, 146, 831-837. http://dx.doi.org/10.1530/eje.0.1460831

[4] Ramirez-Torres, M.A. (2005) Gestational Diabetes Mellitus, Experience at a Third Level Hospital. Ginecología y Obstetricia de México, 73, 484-491.

[5] Moses, R.G. (2010) New Consensus Criteria for GDM: Problem Solved or a Pandora’s Box? Diabetes Care, 33, 690691. http://dx.doi.org/10.2337/dc09-2306

[6] Di Cianni, G., Seghieri, G., Lencioni, C., Cuccuru, I., Anichini, R., De Bellis, A., et al. (2007) Normal Glucose Tolerance and Gestational Diabetes Mellitus: What Is in between? Diabetes Care, 30, 1783-1788. http://dx.doi.org/10.2337/dc07-0119

[7] International Association of Diabetes and Pregnancy Study Groups Consensus Panel (2010) International Association of Diabetes and Pregnancy Study Groups Recommendations on the Diagnosis and Classification of Hyperglycemia in Pregnancy. Diabetes Care, 33, 676-682. http://dx.doi.org/10.2337/dc09-1848

[8] Waugh, N., Royle, P., Clar, C., Henderson, R., Cummins, E., Hadden, D., et al. (2010) Screening for Hyperglycaemia in Pregnancy: A Rapid Update for the National Screening Committee. Health Technological Assessment, 14, 1-183.

[9] Ministry of Health (2009) Clinical Practice, Diagnosis, and Treatment Guidelines for Diabetes in Pregnancy.

[10] HAPO Study Cooperative Research Group (2008) Hyperglycemia and Adverse Pregnancy Outcomes. New England Journal of Medicine, 358, 1991-2002. http://dx.doi.org/10.1056/NEJMoa0707943

[11] Mestzger, B.E. and Coustan, D.R. (1998) Summary and Recommendations of the Fourth International Workshop-Conference on Gestational Diabetes Mellitus. The Organizing Committee. Diabetes Care, 21, B161-B167.

[12] Basevi, V., Di Mario, S., Morciano, C., Nonino, F. and Magrini, N. (2011) Commnent on: American Diabetes Association. Standards of Medical Care in Diabetes-2011. Diabetes Care, 34, S11-S61. http://dx.doi.org/10.2337/dc11-S011

[13] American College of Obstetricians and Gynecologists (2011) Committee Opinion No. 504. Screening and Diagnosis of Gestational Diabetes Mellitus. Obstetrics \& Gynecology, 118, 751-753. http://dx.doi.org/10.1097/AOG.0b013e3182310cc3

[14] Reyes-Muñoz, E., Parra, A., Castillo-Mora, A. and Ortega-González, C. Effect of Diagnostic Criteria of the International Association of Diabetes and Pregnancy Study Groups on the Prevalence of Gestational Diabetes Mellitus in Urban Mexican Women: A Cross-Sectional Study. Endocrine Practice, 18, 146-151. http://dx.doi.org/10.4158/EP11167.OR

[15] Ortega-González, C., Ballesteros, A., Casanueva, E., Fonseca, T., Cacique, E. and Parra, A. (2008) Searching for Alternative Methods and Diagnosing Gestational Diabetes Mellitus in a Mexican Urban Population. Medical Science Monitor, 148, CR598-CR603.

[16] Nolasco-Blé, A.K., Hernandez-Herrera, R.J. and Ramos-González, R.M. (2012) Perinatal Outcomes in Pregnancies of Advanced Maternal Age. Ginecología y Obstetricia de México, 80, 270-275.

[17] Schaefer-Graf, U., Kjos, S., Kilavuz, O., Plagemann, A., Brauer, M., Dudenhausen, J., et al. (2003) Determinants of Fetal Growth at Different Periods of Pregnancies Complicated by Gestational Diabetes Mellitus or Impaired Glucose Tolerance. Diabetes Care, 26, 193-198. http://dx.doi.org/10.2337/diacare.26.1.193

[18] Landon, M.B., Spong, C.Y., Thom, E., Carpenter, M.W., Ramin, S.M., Casey, B., et al. (2009) A Multicenter, Randomized Trial of Treatment for Mild Gestational Diabetes. New England Journal of Medicine, 361, 1339-1348. http://dx.doi.org/10.1056/NEJMoa0902430 